\title{
Grey FNN control and robustness design for practical nonlinear systems
}

DOI:10.36909/jer.11273

\author{
ZY Chen*, Yahui Meng**, Ruei-yuan Wang***, Timothy Chen****
}

* Guangdong University of Petrochem Technol, Sch Sci, Maoming 525000, Peoples R China,

** Guangdong University of Petrochem Technol, Sch Sci, Maoming 525000, Peoples R China, Email: mengyahui@gdupt.edu.cn, the 1st corresponding author

*** Guangdong University of Petrochem Technol, Sch Sci, Maoming 525000, Peoples R China, Email: rueiyuan@gmail.com, the 2nd corresponding author

**** Division of Engineering and Applied Science, California Institute of Technology, Pasadena, CA 91125

Email t13929751005@gmail.com, the $3^{\text {rd }}$ corresponding author

\begin{abstract}
To ensure asymptomatic stability and improve vehicle ride comfort, this paper develops a fuzzy neural network (FNN) based on the evolved bat algorithm (EBA) to design adaptive backstepping controllers with gray signal predicators. A recoil method is used to evaluate the nonlinearity of the controlled systems and to derive the control law which is evolved for the tracking of the signals. A group of grey differential equations are applied for the grey model (GM) $(n, h)$, which is an active model where $h$ is the number of considered variables and $n$ is the order of the grey differential equations. In the article, the Discrete GM (2.1) is used to obtain the advanced motion of the nonlinear system, so that the command controller can prove the Lyapunov stability and feasibility of the entire scheme through the Lyapunov-like lemma. The controller design criteria are demonstrated for mechanical elastic wheels (MEW) to establish a viable mathematical framework for the new wheels.
\end{abstract}


Keywords: evolved control, Lyapunov energy function, DGM (2.1), adaptive backstepping, neural network.

\section{I . Introduction}

With the rapid growth of the automobile industry, automobile safety has also been significantly improved (Singh et al., 2019; Cisija-Kobilica et al., 2019). More and more automobile manufacturers car company that makes automobile are beginning to pay attention to removing noise, vibration and drive irregularities (Cho et al,2017; Andert et al,2017; Charfeddine and Jerbi, 2021). So far, people have made great efforts have been made to improve driving stillness, including the development of active steering, better steerable steering systems and steering motors (Xiangyang et al,2017;Zhang and Wang et al,2017). As one of the most important components of the vehicle, the chassis plays an important role in ameliorating driving vibration stillness and improving performance (Moreno Ramírez et al, 2018; Zhang et al, 2018; Kilicaslan, 2018; Kawamoto et al, 2018). Many researchers began to consider artificial intelligence control methodology for the systems.

Takagi and Sugeno proposed a new fuzzy model, Takagi-Sugeno fuzzy model in 1985. Also, grey system theory is a good choice for a control system. The grey model, denoted by GM (n,h) in grey system theory, is a dynamic model which consists of a group of grey differential equations, where $\mathrm{n}$ is the order of the grey differential equations and $h$ is the number of considered variables. The grey models play an important role in sequence (series) forecasting problems in grey system theory. Among all the GM $(\mathrm{n}, \mathrm{h})$ models, the most commonly utilized is GM $(1,1)$ model because of the simplicity of the modeling process and, especially, the forecasting accuracy. In practical nonlinear application, however, it is not easy to know the precise data for the system input and output due to the complexity of the control law or the time delay caused by the signal transmission of the system, causing the control quantity calculated by the control law to fail to respond to the control system.

The requirements of the current state of the system cannot achieve good control. For this type of 
situation, gray predictive control can be used to find the changing law of system behavior development, and to predict the state of the system. This is the so-called advanced control to compensate for the time delay of the controlled systems. Because of the strong merits of the T-S fuzzy model, the nonstationary control for T-S fuzzy Markovian switching systems is presented for practical application. The finite-time stabilization of the T-S fuzzy semi-Markov switching systems has also been proven robust for a sampled-data control approach (for more details, please see (Chen et al, 2021).

In the above control theories, a back-propagation neural network method is believed suitable to generate the proper drive signal after learning the dynamics of the whole system. This method can be described as follows. First, we use the neural network to learn the dynamic modeling of the unknown nonlinear system. The neural network can be interpreted based on the relationship between the input and output of the whole system. Then, the neural network generates the appropriate drive signal to achieve the desired performance. This method is called inverse modeling. It is not necessary to analyze the mathematical model of the system. Through many iterations we can obtain good performance in spite of the higher order and nonlinear system.

The nonlinear structure of the network is as close as possible to that of the neural network. In order to simplify the control problem, the LDI (Linear Differential Inclusion) notation is used to study the stability of large-scale nonlinear proportional systems. As we know, the evolutionary bat algorithm (EBA) can be used to solve the numerical optimization problem of swarm intelligence, improving the accuracy to find the best short-range solution, and shorten the calculation process.

Generally, the work of control strategies to improve vehicle riding comfort (especially active suspension) for such wheels is rarely concentrated on the control strategy. It is necessary to consider nonlinear systems and perform some operations in this area with certainty. The gray DGM model (2.1) is particularly appropriate for be writing no monotonic vibration sequences and it was widely applied in signal analyses (Shao and Su, 2012; Huang et al, 2016; Deshpande et al, 2014; Tsai et al, 
2011; Chen, 2014; Chen et al, 2020). Therefore, in this study, the gray DGM model (2.1) is used to provide predictive information and increase the power of the system controller can make powerful to make the best for suspension control system (Chen et al, 2022).

In short, the focus of this article is to announce the new features of elastic mechanics to avoid wheel failure, and to design a more efficient steering wheel to match the new wheels with suspension. In order to reflect the real situation, we consider the speed and uncertainty of the wheel and the suspension. To identify the continuity of best-in-class removal methods, gray signals are used to obtain future outputs. Finally, the statistical analysis evaluated the effectiveness and reliability of the proposed control model.

The rest of this paper is organized as follows. The Section I describes the description of the system. In case of model errors, section I provides sufficient conditions for asymptomatic stability of nonlinear system. A numerical example with simulation is given to show the feasibility of our method in Chapter IV, and some of the concluding remarks are given to conclude the paper.

\section{System Description}

Let $x^{(0)}$ be a non-negative original data sequence,

$$
x^{(0)}=\left(x^{(0)}(1), x^{(0)}(2), \Lambda, x^{(0)}(n)\right)
$$

and suppose that $x^{(1)}$ is the first-order AGO sequence,

$$
x^{(1)}=\left(x^{(1)}(1), x^{(1)}(2), \Lambda, x^{(1)}(n)\right) .
$$

Then, by taking AGO on $x^{(0)}, x^{(1)}$ can be obtained by

$$
\begin{aligned}
& x^{(1)}(k)=A G O \circ x^{(0)}=\sum_{m=1}^{k} x^{(0)}(m), \quad k=1,2 \bigwedge n, \\
& x^{(j)}(k)=\sum_{m=1}^{k} x^{(j-1)}(m), \quad k=1,2 \bigwedge n,
\end{aligned}
$$

Contrarily, $x^{(0)}$ can be obtained by taking IAGO on $x^{(1)}$ as 


$$
x^{(0)}=L A G O \circ x^{(1)}= \begin{cases}x^{(1)}(k)-x^{(1)}(k-1), & \text { for } k \geq 2, \\ x^{(1)}(1), & \text { for } k=1 .\end{cases}
$$

Let $z^{(1)}$ be the sequence obtained by applying MEAN generating operation, for simplify denoted by MEAN, to $x^{(1)}$ as

$$
z^{(1)}=\left(z^{(1)}(2), z^{(1)}(3), \Lambda, z^{(1)}(n)\right)
$$

Then,

$$
z^{(1)}=\operatorname{MEAN} \quad \circ\left\{x^{(1)}(k), x^{(1)}(k-1)\right\} .
$$

The data generating operations are viewed as the first step in building a grey model (GM). Especially, AGO operation can be transformed the disorderly row data to the regular sequence, and find out the interval or hidden regular property of the original sequence, and then it also can weaken the randomness of the irregular sequence.

The Grey Difference Equation of GM(1,1)

$$
x^{(0)}(k)+a z^{(1)}(k)=u,
$$

is called a grey difference equation of $\operatorname{GM}(1,1)$, where $a$ and $u$ are called the development coefficient and the grey input, respectively. And the corresponding whitening equation is represented as follows

$$
\begin{aligned}
& \frac{d y^{(1}(t)}{d t}+a y^{(1}(t)=u \\
& y^{(0)}(k)=y^{(1)}(k)-y^{(1)}(k-1) \approx \frac{d y^{(1)}(t)}{d t} \\
& z^{(1)}(k)=y^{(1)}(t)
\end{aligned}
$$

Proof. Assume

$$
x^{(0)}(k)+a z^{(1)}(k)=k b
$$

For $Y=B a$, which gives the error sequence $\varepsilon=Y-B a$. Let 


$$
\begin{gathered}
S=\varepsilon^{T} \varepsilon=(Y-B a)^{T}(Y-B a)=\sum_{k=2}^{n}\left(x^{(0)}(k)+a z^{(1)}(k)-b k\right)^{2} . \\
\left\{\begin{array}{l}
\frac{\partial s}{\partial a}=2 \sum_{k=2}^{n}\left(x^{(0)}(k)+\mathrm{az}^{(1)}(k)-b k\right) z^{(1)}(k)=0 \\
\frac{\partial s}{\partial b}=-2 k \sum_{k=2}^{n}\left(x^{(0)}(k)+a z^{(1)}(k)-b k\right)=0 .
\end{array}\right.
\end{gathered}
$$

\begin{tabular}{|c|c|}
\hline Symbol & Description \\
\hline M & Mass of car body \\
\hline $\mathrm{mn}, \mathrm{m} 2$ & Mass of front or rear MEW \\
\hline$z$ & Vertical displacement of car body \\
\hline 21,22 & Vertical displacement of front or rear MEW \\
\hline la & Moment of inertia around the centroid \\
\hline h & Pitch angle of car body \\
\hline$a, b$ & Distances between centroid and front or rear suspensions \\
\hline $\mathrm{Fin}, \mathrm{Fiz}$ & Spring force of front or rear suspension \\
\hline$F_{c 1}, F a$ & Damping force of front or rear suspension \\
\hline us, uz & Forces generated by front or rear actuator \\
\hline $\mathrm{Fu}, \mathrm{Fu}$ & Tire forces of front or rear MEW \\
\hline$v$ & Speed of vehicle \\
\hline$q_{1}, q_{2}$ & Road profiles \\
\hline
\end{tabular}

Table 1 Nomenclature.

The original state, as shown in Table 1, is described as the position without forces on the suspension and wheels. The direction and dynamics of the axis can describe the following (Chen, 2021):

$$
\left\{\begin{array}{l}
\square \\
x_{1}=x_{2} \\
x_{2}=\frac{1}{M}\left[-F_{k 1}-F_{c 1}+u_{1}-F_{k 2}-F_{c 2}+u_{2}-M g\right] \\
x_{3}=x_{4} \\
x_{4}=\frac{1}{l y}\left[F_{k 1} a+F_{c 1} a-u_{1} a-F_{k 2} b-F_{c 2} b+u_{2} b\right] . \\
x_{5}=x_{6} \\
x_{6}=\frac{1}{m_{1}}\left[F_{k 1}+F_{c 1}-u_{1}+F_{t 1}-m_{1} g\right] \\
x_{7}=x_{8} \\
x_{8}=\frac{1}{m_{2}}\left[F_{k 2}+F_{c 2}-u_{2}+F_{t 2}-m_{2} g\right]
\end{array}\right.
$$

So far, we consider the nonlinear distance between suspension and water wave, and the active suspension system with water wave is modeled. In the next part, we consider the suspension controller consists of an adaptive backstep control law, an ideal suspension motion generator and a gray signal predictor, which controls the suspension motion to check the ideal state, and the signal predictor is adjusted again as expected. state projected by control law. The control structure is 
shown in Figure 1. For stable body, let us assume that there is ideal suspension movement. If the true vertical and pitching motion of the vehicle can track the required movement, the comfort of movement can be guaranteed. First, we describe the monitoring error. Lyapunov's error form can describe the following.

$$
\begin{aligned}
V\left(e_{1}, e_{3}, \delta_{2}, \delta_{4}\right) & =e_{1} e_{1}+e_{3} e_{3}+\delta_{2} \delta_{2}+\delta_{4}{ }^{\square}=e_{1}\left(x_{1 d}-x_{2}\right)+e_{3}\left(x_{3 d}-x_{4}\right)+\delta_{2} \stackrel{\square}{\delta}_{2}+\delta_{4} \stackrel{\square}{\delta}_{4} \\
& =e_{1}\left[x_{1 d}^{\square}-\left(x_{1 d}+k_{1} e_{1}-\delta_{2}\right)\right]+e_{3}\left[x_{3 d}-\left(x_{3 d}+k_{3} e_{3}-\delta_{4}\right)\right]+\delta_{2} \stackrel{\square}{\delta}_{2}+\delta_{4} \stackrel{\square}{\delta_{4}} \\
& =-k_{1} e_{1}^{2}-k_{3} e_{3}^{2}+\delta_{2}\left(e_{1}+\stackrel{\delta}{2}_{2}\right)+\delta_{4}\left(e_{3}+\delta_{4}\right)
\end{aligned}
$$

where $e_{1}, e_{3}$ denote the actual vertical motion compared with ideal suspension motion, and pitch motion of vehicle compared to the ideal suspension motion. $\delta$ is the differential form of $e$.

Consider an NN model, $\mathrm{S}$ layers with $R^{q}(\mathrm{q}=1,2, \cdots, \mathrm{S})$ neurons for each layer, in which $x(k) \sim x(k-m+1)$ are the state variables and $u(k) \sim u(k-n+1)$ are the input variables.

The number of the layer is appended as a superscript to the names of the variables. Thus, the weight matrix of the qth layer is written as $W^{q}$. Then the final output of the NN model can be inferred as follows:

$$
x(k+1)=\psi^{S}\left(W^{S} \times \Psi^{S-1}\left(W^{S-1} \times \Psi^{S-2}\left(\ldots \ldots \Psi^{2}\left(W^{2} \times \Psi^{1}\left(W^{1} \times Z(k)\right)\right) \ldots \ldots\right)\right)\right)
$$

Furthermore, based on the interpolation method and equation, we can obtain

$$
\begin{aligned}
& x(k+1)=\left\{\sum _ { j _ { 1 } ^ { s } = 1 } ^ { 2 } \cdots \sum _ { j _ { R ^ { s } } ^ { s } = 1 } ^ { 2 } h _ { j _ { 1 } ^ { s } } ^ { S } ( k ) \ldots h _ { j _ { R ^ { s } } ^ { s } } ^ { S } ( k ) G ( v ^ { S } , \psi ^ { S } ) \left(W^{S} \times\left[\cdots \cdots \left[\sum_{j_{1}^{2}=1}^{2} \cdots \sum_{j_{R^{2}}^{2}=1}^{2} h_{j_{1}^{2}}^{2}(k) \cdots h_{j_{R^{2}}^{2}}^{2}(k)\right.\right.\right.\right. \\
&\left.\left.\left.\left.\cdot G\left(v^{2}, \Psi^{2}\right)\left(W^{2} \times\left[\sum_{j_{1}^{1}=1}^{2} \cdots \sum_{j_{R^{1}=1}^{1}}^{2} h_{j_{1}^{1}}^{1}(k) \cdots h_{j_{R^{1}}^{1}}^{1}(k) G\left(v^{1}, \Psi^{1}\right)\left(W^{1} \times Z(k)\right)\right]\right)\right] \cdots \cdot \cdot\right]\right)\right\} \\
&=\sum_{v} h_{v}(k) J_{v}(W, \psi) Z(k),
\end{aligned}
$$

The dynamics of the NN model can be rewritten as the following LDI representation:

$$
x(k+1)=\sum_{i=1}^{\phi} h_{i}(k) J_{i} Z(k)=\sum_{i=1}^{\phi} h_{i}(k)\left\{A_{i} x(k)+B_{i} u(k)\right\},
$$


and $J_{i}$ is a constant matrix with the appropriate dimension associated with $J_{v}(W, \Psi)$.

The final output of this fuzzy controller is inferred as follows:

$$
u(k)=-\frac{\sum_{j=1}^{J} w_{j}(k) K_{j} x(k)}{\sum_{j=1}^{J_{l}} w_{j}(k)}=-\sum_{j=1}^{J_{l}} \bar{h}_{j}(k) K_{j} x(k),
$$

with $w_{j}(k)=\prod_{\mu=1}^{p} M_{j \mu}\left(x_{\mu}(k)\right), \quad \bar{h}_{j}(k)=\frac{w_{j}(k)}{\sum_{j=1}^{J_{l}} w_{j}(k)}$

in which $M_{j \mu}\left(x_{\mu}(k)\right)$ is the grade of membership of $x_{\mu}(k)$ in $M_{j \mu}$. In this study, it is also assumed that $w_{j}(k) \geq 0$, and $\sum_{j=1}^{J_{l}} w_{j l}(k)>0$ for all $k$. Therefore, $\bar{h}_{j}(k) \geq 0$ and $\sum_{j=1}^{J_{l}} \bar{h}_{j}(k)=1$ for all $k$.

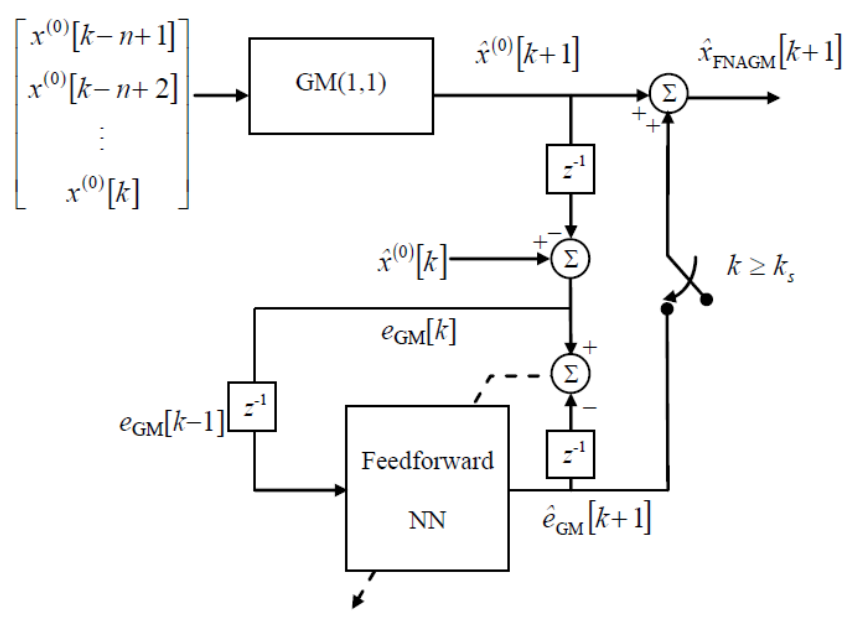

Fig. 1 LDI based grey prediction control model

\section{Evolved NN modeling and stability of frey signal predictor}

The desired ideal motion is obtained by good hybrid damping control.The virtual damping coefficient is given as the control signal.

Watch the law of control, which includes monitoring errors and rate. If $\mathrm{B}=[\mathrm{p}, \mathrm{q} 1, \mathrm{greyDGM}(21)$ model can describe the following.

$$
\alpha^{(1)} x^{(0)}+p x^{(0)}(k)=q, \quad B h=I
$$




$$
B=\left[\begin{array}{cc}
-x^{(0)}(2) & 1 \\
-x^{(0)}(3) & 1 \\
\vdots & \vdots \\
-x^{(0)}(n) & 1
\end{array}\right], Y=\left[\begin{array}{c}
\alpha^{(1)} x^{(0)}(2) \\
\alpha^{(1)} x^{(0)}(3) \\
\vdots \\
\alpha^{(1)} x^{(0)}(n)
\end{array}\right]=\left[\begin{array}{c}
x^{(0)}(2)-x^{(0)}(1) \\
x^{(0)}(3)-x^{(0)}(2) \\
\vdots \\
x^{(0)}(n)-x^{(0)}(n-1)
\end{array}\right]
$$

Furthermore, the least square method is used to obtain

$\theta=\left[\begin{array}{l}a \\ u\end{array}\right]=\left(B^{T} B\right)^{-1} B^{T} Y$

where

$$
a=\frac{A_{1} A_{2}-(n-1) A_{3}}{(n-1) A_{4}-A_{1}^{2}}, u=\frac{A_{2} A_{4}-A_{1} A_{3}}{(n-1) A_{4}-A_{1}^{2}}
$$

After assumption, the true value of the signal is measured in time. Due to the effect of random excitation on the levitation motion, we consider equation (8).

$$
x^{(0)}(n+1)=\left\{\begin{array}{lll}
x^{(0)}(n+1) & \left|x^{(0)}(n+1)-x^{(0)}(n)\right| \leq 5\left|x^{(0)}(n)-x^{(0)}(n-1)\right| \\
x^{(0)}(n) & \left|x^{(0)}(n+1)-x^{(0)}(n)\right|>5\left|x^{(0)}(n)-x^{(0)}(n-1)\right|
\end{array}\right.
$$

If the matrix $\boldsymbol{P}$ and the constant $k$ exist in inequalities:

$$
\boldsymbol{H}_{i j}^{T} \boldsymbol{P} \boldsymbol{H}_{i j}-\boldsymbol{P}<0, \quad(1+\kappa) \boldsymbol{H}_{i j}^{T} \boldsymbol{P} \boldsymbol{H}_{i j}-\boldsymbol{P}+\left(1+\kappa^{-1}\right) \lambda_{\max }(\boldsymbol{P}) \boldsymbol{H}_{q}^{T} \boldsymbol{H}_{q}<0
$$

are satisfied for $i=1,2, \cdots, \phi ; j=1,2, \cdots, l$, then the system is asymptotically stable.

Proof:

Let the Lyapunov function for the nonlinear system be defined as

$$
V(k)=x(k)^{T} P x(k)
$$

Where $P$ is a positive definite matrix. We then evaluate the backward difference of $V(k)$ on the trajectories to get

$$
\begin{gathered}
\Delta V(k)=V(k+1)-V(k)=x(k+1)^{T} \operatorname{Px}(k+1)-x(k)^{T} \operatorname{Px}(k) \\
=\left\{\sum_{i=1}^{\phi} \sum_{j=1}^{l} h_{i}(k) \bar{h}_{j}(k) H_{i j} x(k)+e(k)\right\}^{T} P\left\{\sum_{i=1}^{\phi} \sum_{j=1}^{l} h_{i}(k) \bar{h}_{j}(k) H_{i j} x(k)+\mathrm{e}(x)\right\}-x(k)^{T} P x(k)
\end{gathered}
$$

Let 


$$
\sum_{i=1}^{\phi} \sum_{j=1}^{l} \sum_{\alpha=1}^{\phi} \sum_{\beta=1}^{l} h_{i}(k) \bar{h}_{j}(k) h_{\alpha}(k) \bar{h}_{\beta}(k) x(k)^{T}\left\{H_{i j}^{T} P H_{\alpha \beta}-P\right\} x(k)=m_{1}+m_{2}+m_{3}+m_{4}
$$

Where

$$
\begin{aligned}
& m_{1}=\sum_{i=\alpha}^{\phi} \sum_{j=\beta}^{l} h_{i}^{2}(k) \bar{h}_{j}^{2}(k) x(k)^{T}\left(H_{i j}^{T} P H_{i j}-P\right) x(k), \\
& m_{2} \equiv \sum_{i \neq \alpha}^{\phi} \sum_{j=\beta}^{l} h_{i}(k) h_{\alpha}(k) \bar{h}_{j}^{2}(k) \boldsymbol{x}(k)^{T}\left(\boldsymbol{H}_{i j}^{T} \boldsymbol{P} \boldsymbol{H}_{\alpha j}-\boldsymbol{P}\right) \boldsymbol{x}(k) \\
& =\sum_{i<\alpha}^{\phi} \sum_{j=\beta}^{l} h_{i}(k) h_{\alpha}(k) \bar{h}_{j}^{2}(k) \boldsymbol{x}(k)^{T}\left\{\boldsymbol{H}_{i j}^{T} \boldsymbol{P} \boldsymbol{H}_{\alpha j}+\boldsymbol{H}_{\alpha j}^{T} \boldsymbol{P} \boldsymbol{H}_{i j}-2 \boldsymbol{P}\right\} \boldsymbol{x}(k), \\
& m_{3} \equiv \sum_{i=\alpha}^{\phi} \sum_{j \neq \beta}^{l} h_{i}^{2}(k) \bar{h}_{j}(k) \bar{h}_{\beta}(k) \boldsymbol{x}(k)^{T}\left(\boldsymbol{H}_{i j}^{T} \boldsymbol{P} \boldsymbol{H}_{i \beta}-\boldsymbol{P}\right) \boldsymbol{x}(k) \\
& =\sum_{i=\alpha}^{\phi} \sum_{j<\beta}^{l} h_{i}^{2}(k) h_{j}(k) h_{\beta}(k) \boldsymbol{x}(k)^{T}\left\{\boldsymbol{H}_{i j}^{T} \boldsymbol{P} \boldsymbol{H}_{i \beta}+\boldsymbol{H}_{i \beta}^{T} \boldsymbol{P} \boldsymbol{H}_{i j}-2 \boldsymbol{P}\right\} \boldsymbol{x}(k), \\
& m_{4} \equiv \sum_{i \neq \alpha}^{\phi} \sum_{j \neq \beta}^{l} h_{i}(k) \bar{h}_{j}(k) h_{\alpha}(k) \bar{h}_{\beta}(k) \boldsymbol{x}(k)^{T}\left(\boldsymbol{H}_{i j}^{T} \boldsymbol{P} \boldsymbol{H}_{\alpha \beta}-\boldsymbol{P}\right) \boldsymbol{x}(k) \\
& =\sum_{i<\alpha}^{\phi} \sum_{j<\beta}^{l} h_{i}(k) h_{j}(k) h_{\alpha}(k) h_{\beta}(k) \boldsymbol{x}(k)^{T}\left\{\boldsymbol{H}_{i j}^{T} \boldsymbol{P} \boldsymbol{H}_{\alpha \beta}+\boldsymbol{H}_{\alpha \beta}^{T} \boldsymbol{P} \boldsymbol{H}_{i j}-2 \boldsymbol{P}\right\} \boldsymbol{x}(k) .
\end{aligned}
$$

Therefore, we have

$$
\begin{aligned}
m_{2}= & \sum_{i<\alpha}^{\phi} \sum_{j=\beta}^{l} h_{i}(k) h_{\alpha}(k) \bar{h}_{j}^{2}(k) \boldsymbol{x}(k)^{T}\left\{-\left[\boldsymbol{H}_{i j}-\boldsymbol{H}_{\alpha j}\right]^{T} \boldsymbol{P}\left[\boldsymbol{H}_{i j}-\boldsymbol{H}_{\alpha j}\right]+\boldsymbol{H}_{i j}^{T} \boldsymbol{P} \boldsymbol{H}_{i j}-\boldsymbol{P}\right. \\
& \left.+\boldsymbol{H}_{\alpha j}^{T} \boldsymbol{P} \boldsymbol{H}_{\alpha j}-\boldsymbol{P}\right\} \boldsymbol{x}(k)<0 \quad \text { for } i<\alpha \leq \phi ; j=\beta=1, \cdots, l .
\end{aligned}
$$

In similar fashion

$$
m_{3}<0 \text { and } m_{4}<0 \text {. }
$$

Substituting (A.4) and (A.5) into (A.3) yields

$$
\begin{aligned}
& \sum_{i=1}^{\phi} \sum_{j=1}^{l} \sum_{\alpha=1}^{\phi} \sum_{\beta=1}^{l} h_{i}(k) \bar{h}_{j}(k) h_{\alpha}(k) \bar{h}_{\beta}(k) \boldsymbol{x}(k)^{T}\left\{\boldsymbol{H}_{i j}^{T} \boldsymbol{P} \boldsymbol{H}_{\alpha \beta}-\boldsymbol{P}\right\} \boldsymbol{x}(k) \\
\leq & \sum_{i=1}^{\phi} \sum_{j=1}^{l} h_{i}(k) \bar{h}_{j}(k) \boldsymbol{x}(k)^{T}\left(\boldsymbol{H}_{i j}^{T} \boldsymbol{P} \boldsymbol{H}_{i j}-\boldsymbol{P}\right) \boldsymbol{x}(k) .
\end{aligned}
$$

From (A.6) and (A.2), we have

$$
\begin{aligned}
\Delta V(k) \leq & \sum_{i=1}^{\phi} \sum_{j=1}^{l} h_{i}(k) \bar{h}_{j}(k) \boldsymbol{x}(k)^{T}\left(\boldsymbol{H}_{i j}^{T} \boldsymbol{P} \boldsymbol{H}_{i j}-\boldsymbol{P}\right) \boldsymbol{x}(k) \\
& +\sum_{i=1}^{\phi} \sum_{j=1}^{l} h_{i}(k) \bar{h}_{j}(k)\left\{\boldsymbol{x}(k)^{T} \boldsymbol{H}_{i j}^{T} \boldsymbol{P e}(k)+\boldsymbol{e}(k)^{T} \boldsymbol{P} \boldsymbol{H}_{i j} \boldsymbol{x}(k)\right\}+\boldsymbol{e}(k)^{T} \boldsymbol{P e}(k) .
\end{aligned}
$$

Since $\boldsymbol{P}$ is a positive definite matrix, it follows that

$$
\left(\kappa^{\frac{1}{2}} \boldsymbol{H}_{i j} \boldsymbol{x}(k)-\kappa^{-\frac{1}{2}} \boldsymbol{e}(k)\right)^{T} \boldsymbol{P}\left(\kappa^{\frac{1}{2}} \boldsymbol{H}_{i j} \boldsymbol{x}(k)-\kappa^{-\frac{1}{2}} \boldsymbol{e}(k)\right) \geq 0
$$




$$
\begin{aligned}
& \Rightarrow \kappa \boldsymbol{x}(k)^{T} \boldsymbol{H}_{i j}^{T} \boldsymbol{P} \boldsymbol{H}_{i j} \boldsymbol{x}(k)-\boldsymbol{x}(k)^{T} \boldsymbol{H}_{i j}^{T} \boldsymbol{P e}(k)-\boldsymbol{e}(k)^{T} \boldsymbol{P} \boldsymbol{H}_{i j} \boldsymbol{x}(k)+\kappa^{-1} \boldsymbol{e}(k)^{T} \boldsymbol{P e}(k)^{T} \geq 0 \\
& \Rightarrow \boldsymbol{x}(k)^{T} \boldsymbol{H}_{i j}^{T} \boldsymbol{P e}(k)+\boldsymbol{e}(k)^{T} \boldsymbol{P} \boldsymbol{H}_{i j} \boldsymbol{x}(k) \leq \boldsymbol{\kappa} \boldsymbol{x}(k)^{T} \boldsymbol{H}_{i j}^{T} \boldsymbol{P} \boldsymbol{H}_{i j} \boldsymbol{x}(k)+\boldsymbol{\kappa}^{-1} \boldsymbol{e}(k)^{T} \boldsymbol{P e}(k)^{T} .
\end{aligned}
$$

Therefore, we obtain

$$
\begin{aligned}
\Delta V(k) & \leq \sum_{i=1}^{\phi} \sum_{j=1}^{l} h_{i}(k) \bar{h}_{j}(k) \boldsymbol{x}(k)^{T}\left\{\boldsymbol{H}_{i j}^{T} \boldsymbol{P} \boldsymbol{H}_{i j}-\boldsymbol{P}\right\} \boldsymbol{x}(k) \\
& \leq \sum_{i=1}^{\phi} \sum_{j=1}^{l} h_{i}(k) \bar{h}_{j}(k) \boldsymbol{x}(k)^{T}\left\{(1+\kappa) \boldsymbol{H}_{i j}^{T} \boldsymbol{P} \boldsymbol{H}_{i j}-\boldsymbol{P}\right\} \boldsymbol{x}(k)+\left(1+\kappa^{-1}\right) \lambda_{\max }(\boldsymbol{P}) \boldsymbol{e}(k)^{T} \boldsymbol{e}(k) .(\mathrm{A} .8)
\end{aligned}
$$

From (A.8) we can get

$$
\Delta V(k) \leq \sum_{i=1}^{\phi} \sum_{j=1}^{l} h_{i}(k) \bar{h}_{j}(k) \boldsymbol{x}(k)^{T}\left\{(1+\kappa) \boldsymbol{H}_{i j}^{T} \boldsymbol{P} \boldsymbol{H}_{i j}-\boldsymbol{P}+\left(1+\kappa^{-1}\right) \lambda_{\max }(\boldsymbol{P}) \boldsymbol{H}_{q}^{T} \boldsymbol{H}_{q}\right\} \boldsymbol{x}(k) .
$$

The closed-loop nonlinear system is asymptotically stable.

Based on Eqs. (5-6), we have

$$
\begin{gathered}
x(k+1)=\sum_{i=1}^{\phi} \sum_{j=1}^{l} h_{i}(k) \bar{h}_{j}(k)\left(A_{i}+B_{i} K_{j}\right) x(k)+\left\{F(x(k))-\sum_{i=1}^{\phi} \sum_{j=1}^{l} h_{i}(k) \bar{h}_{j}(k)\left(A_{i}+B_{i} K_{j}\right) x(k)\right\} \\
=\sum_{i=1}^{\phi} \sum_{j=1}^{l} h_{i}(k) \bar{h}_{j}(k) H_{i j} x(k)+e(k)
\end{gathered}
$$

where

$$
H_{i j}=A_{i}+B_{i} K_{j}, \quad e(k)=\left\{F(x(k))-\sum_{i=1}^{\phi} \sum_{j=1}^{l} h_{i}(k) \bar{h}_{j}(k)\left(A_{\mathrm{i}}+B_{i} K_{j}\right) x(k)\right\}
$$

which denotes the modeling error between the closed-loop nonlinear system and the closed-loop NN system.

Suppose that there exits a bounding matrix $\Delta H_{i j}$ such that

$$
\|e(k)\| \leq\left\|\sum_{i=1}^{\phi} \sum_{j=1}^{l} h_{i}(k) \bar{h}_{j}(k) \Delta H_{i j} x(k)\right\|
$$

for all trajectories $x(k)$ and the bounding matrix $\Delta H_{i j}$ can be described by $\square H_{i j}=\delta_{i j} H_{q}$ where $\left\|\delta_{i j}\right\| \leq 1$, for $i=1,2, \ldots, \phi$ and $j=1,2, \ldots, \mid$, and the modeling error is bounded by the specified structured bounding matrix $H_{q}$. 
Therefore, we can obtain

$$
\begin{aligned}
\boldsymbol{e}^{T}(k) \boldsymbol{e}(k) \leq & \left\{\sum_{i=1}^{\phi} \sum_{j}^{l} h_{i}(k) \bar{h}_{j}(k) \Delta \mathbf{H}_{i j} \mathbf{x}(k)\right\}^{T}\left\{\sum_{i=1}^{\phi} \sum_{j}^{l} h_{i}(k) \bar{h}_{j}(k) \Delta \mathbf{H}_{i j} \mathbf{x}(k)\right\} \\
& =\left\{\sum_{i=1}^{\phi} \sum_{j=1}^{l} h_{i}(k) \bar{h}_{j}(k) \delta_{i j} \boldsymbol{H}_{q} \boldsymbol{x}(k)\right\}^{T}\left\{\sum_{i=1}^{\phi} \sum_{j=1}^{l} h_{i}(k) \bar{h}_{j}(k) \delta_{i j} \boldsymbol{H}_{q} \boldsymbol{x}(k)\right\} \\
& =\boldsymbol{x}(k)^{T} \boldsymbol{H}_{q}^{T}\left[\sum_{i=1}^{\phi} \sum_{j=1}^{l} h_{i}(k) \bar{h}_{j}(k) \delta_{i j}^{T}\right]\left[\sum_{i=1}^{\phi} \sum_{j=1}^{l} h_{i}(k) \bar{h}_{j}(k) \delta_{i j}\right] \boldsymbol{H}_{q} \boldsymbol{x}(k) \\
& \leq\left\|\boldsymbol{H}_{q} \boldsymbol{x}(k)\right\|\left[\sum_{i=1}^{\phi} \sum_{j=1}^{l} h_{i}(k) \bar{h}_{j}(k) \delta_{i j}^{T}\right]\left[\sum_{i=1}^{\phi} \sum_{j=1}^{l} h_{i}(k) \bar{h}_{j}(k) \delta_{i j}\right]\left\|\boldsymbol{H}_{q} \boldsymbol{x}(k)\right\| \\
& \leq\left\|\sum_{i=1}^{\phi} \sum_{j=1}^{l} h_{i}(k) \bar{h}_{j}(k) \delta_{i j}^{T}\right\| \sum_{i=1}^{\phi} \sum_{j=1}^{l} h_{i}(k) \bar{h}_{j}(k) \delta_{i j} \|\left(\boldsymbol{H}_{q} \boldsymbol{x}(k)\right)^{T}\left(\boldsymbol{H}_{q} \boldsymbol{x}(k)\right) \\
& \leq\left\{\boldsymbol{H}_{q} \boldsymbol{x}(k)\right\}^{T}\left\{\boldsymbol{H}_{q} \boldsymbol{x}(k)\right\} .
\end{aligned}
$$

The procedures for determining $\delta_{i j}$ and $\boldsymbol{H}_{q}$ are described by the following simple example.

Assuming that the possible bounds for all elements in $\Delta \boldsymbol{H}_{i j}$ are $\Delta \boldsymbol{H}_{i j}=\left[\begin{array}{ll}\Delta h_{11}^{i j} & \Delta h_{12}^{i j} \\ \Delta h_{21}^{i j} & \Delta h_{22}^{i j}\end{array}\right]$

where $-\varepsilon_{r s} \leq \Delta h_{r s}^{i j} \leq \varepsilon_{r s}$ for some $\varepsilon_{r s}$ and $r, s=1,2$.

One possible description for the bounding matrices $\Delta \boldsymbol{H}_{i j}$ is

$$
\Delta \boldsymbol{H}_{i j}=\left[\begin{array}{cc}
\delta_{11}^{i j} & 0 \\
0 & \delta_{22}^{i j}
\end{array}\right]\left[\begin{array}{ll}
\varepsilon_{11} & \varepsilon_{12} \\
\varepsilon_{21} & \varepsilon_{22}
\end{array}\right]=\delta_{i j} \boldsymbol{H}_{q}
$$

where $-1 \leq \delta_{r r}^{i j} \leq 1$ for $r=1,2$. It is noticed that $\delta_{i j}$ can be chosen by other forms as long as $\left\|\delta_{i j}\right\| \leq 1$

The EBA is hereby proposed with the bat echolocation complex fuzzy system which appears in the practical world. Unlike other intelligence swarm algorithms, the advantage of EBA is good to solve the practical problem. The choice of different media determines search step. In the present 
study, that exists in the practical environment. EBA's function is summarized by some steps:

a. Initialization: distribute artificial reagents by random assignment in the solution chamber.

b. Movement: The artificial sample is moved. Generate a random number and check if it is greater than the fixed heart rate. If the result is positive, you can move the artificial remedy on a random walk

$x_{i}^{t}=x_{i}^{t-1}+D$

where $x_{i}^{t-1}$ the coordinate of the i-th artificial agent at the last iteration; $x_{i}^{t}$ the coordinate of the $\mathrm{i}$-th artificial agent at the $\mathrm{t}$-th iteration; and $\mathrm{D}$ the distance that the artificial agent moves in this iteration.

Thus, $D=\gamma \cdot \Delta T$,

where $\Delta T \in[-1,1]$ a random number. $\gamma$ a constant corresponding to the medium chosen in the experiment; and $\gamma=0.17$ in our experiment because the chosen medium is air.

$x_{i}^{t_{R}}=\beta\left(x_{\text {best }}-x_{i}^{t}\right), \quad \beta \in[0,1]$,

where $x_{\text {best }}$ indicates the coordinate of the near best solution found so far throughout all artificial agents; $\beta$ is a random number; and $x_{i}^{t_{R}}$ represents the new coordinates of the artificial agent after the operation of the random walk process.

c. Evaluation: calculate the suitability of the artificial remedy using a custom suitability function, and update it to the best stored solution.

d. Termination: The termination status is checked to determine if you want to return to STEP 2 or end the program shortly and generate the best solution.

The evaluation criteria for determining the condition of the club are based on a user-defined training function. In this article, the training function is used to get the usual symmetrical positive final matrix and control power to the control.

In general, intelligence swarm algorithms have multiple iterations to find an almost optimal solution. Therefore, the same experiment is chosen to airy in which bats live. The total size indicates solution area for each iteration. We determine the total number and size of possible solutions that are considered sufficient to determine the parameters of the complex cloudy system in the application.

\section{Example}

The fact is that all states in continuous functions of time. When the step is small enough, then we could also conclude that the state is practically unchanged. The Tstep time in DGM (2.1) must 
make control stable and limited. The previously designed controller receives the status of the gray signal predictor to stabilize the motion of the MEW-equipped active suspension. To further test the effectiveness of the controller, a set of movement data is used to test the accuracy of the DGM $(2,1)$ model with a number of signal sequences set to 6 and the step time to $0.001 \mathrm{~s}$. The practical results are listed in Figure 2, where the difference between the exact data and the forecast from the DGM $(2,1)$ is very small. If the data changes drastically, the surface model's tracking error (2.1) increases, but may meet the technical requirements. Therefore, the reliability of DGM (2.1) is guaranteed.

The weight matrices are meant by $\boldsymbol{W}^{1}$ and $\boldsymbol{W}^{2}$. The weights could be given and we obtain

$$
\begin{aligned}
& v_{r}^{1}=W_{1 r}^{1} x(k)+W_{2 r}^{1} x(k-1)+W_{3 r}^{1} u(k), v_{1}^{2}=W_{11}^{2} \mathrm{~T}\left(v_{1}^{1}\right)+W_{21}^{2} \mathrm{~T}\left(v_{2}^{1}\right)+W_{31}^{2} \mathrm{~T}\left(v_{3}^{1}\right), x(k+1)=\mathrm{T}\left(v_{1}^{2}\right) \\
& x(k+1)=\left(h_{11}^{2}(k) g_{1}+h_{12}^{2}(k) g_{2}\right) v_{1}^{2}=\sum_{i=1}^{2} h_{1 i}^{2}(k) g_{i} v_{1}^{2} .
\end{aligned}
$$

Moreover, the NN model can be converted into the following LDI representation by renumbering the matrices:

$$
\boldsymbol{x}(k+1)=\sum_{i=1}^{8} h_{i}(k)\left(\boldsymbol{A}_{i} \boldsymbol{x}(k)+\boldsymbol{B}_{i} u(k)\right)
$$

where

$$
\begin{aligned}
& A_{1}=A_{1 \mathrm{jps}}=A_{2111}=\left[\begin{array}{ll}
0 & 0 \\
1 & 0
\end{array}\right], j, p, s=1,2, A_{2}=A_{2112}=\left[\begin{array}{cc}
-0.6467 & 0.0099 \\
1 & 0
\end{array}\right] \\
& A_{3}=A_{2121}=\left[\begin{array}{cc}
1.5528 & 0.5115 \\
1 & 0
\end{array}\right], A_{4}=A_{2122}=\left[\begin{array}{cc}
-1.3940 & 2.0675 \\
1 & 0
\end{array}\right] \\
& A_{5}=A_{2211}=\left[\begin{array}{cc}
0.9061 & 0.5214 \\
1 & 0
\end{array}\right], A_{6}=A_{2212}=\left[\begin{array}{cc}
-2.0407 & 2.0774 \\
1 & 0
\end{array}\right] \\
& A_{7}=A_{2221}=\left[\begin{array}{cc}
0.1588 & 2.5790 \\
1 & 0
\end{array}\right], A_{8}=A_{2222}=\left[\begin{array}{cc}
-0.4879 & 2.5889 \\
1 & 0
\end{array}\right]
\end{aligned}
$$

The profile of the stepped pavement is shown in Figure 3 (a). As seen in Figs. 3 (b), when road turbulence occurs, the active suspension control force appears within $1 \mathrm{~s}$. In figures 3 (c) and 3 (d), the vertical shift and pitching shift of the vehicle body under of the input step is described. The motion amplitude of the controller with gray signal predictor is smaller than without controller. 


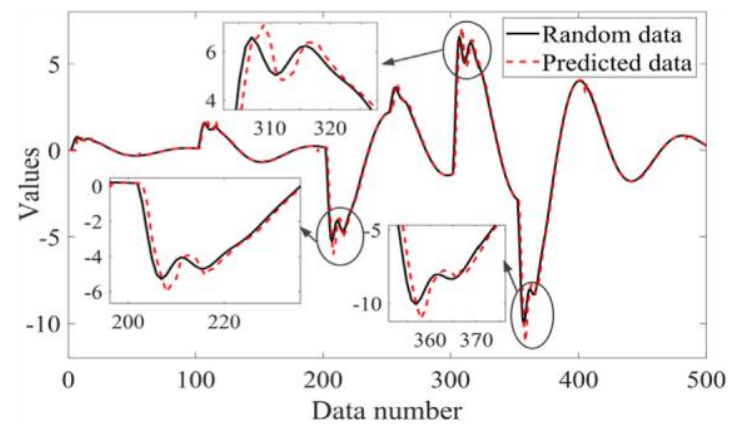

Fig. 2. The random data and proposed fuzzy DGM $(2,1)$ model.

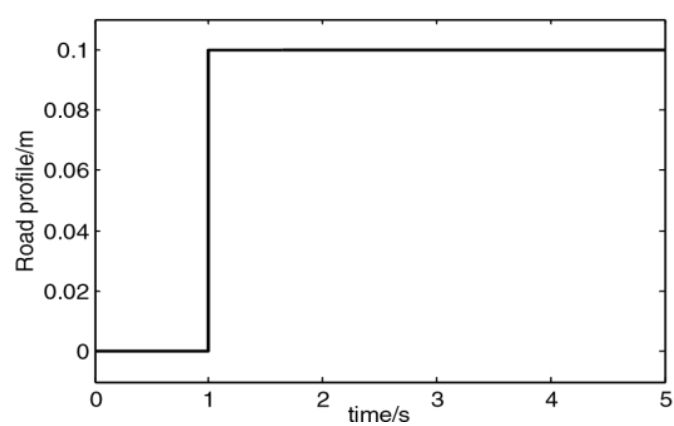

(a) Step road profile

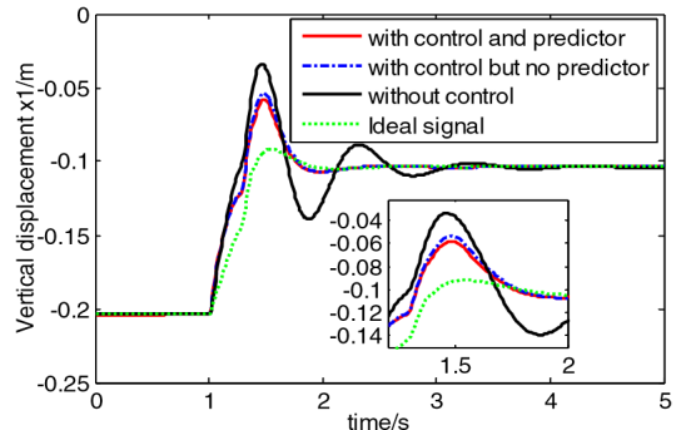

(c) Vertical displacement of car

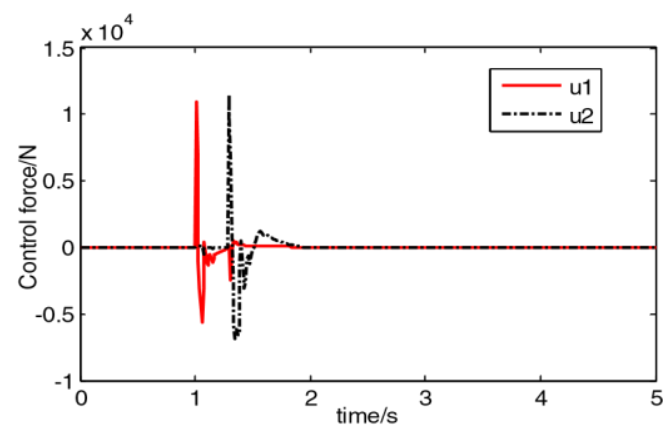

(b) Control signals u1 \&u2

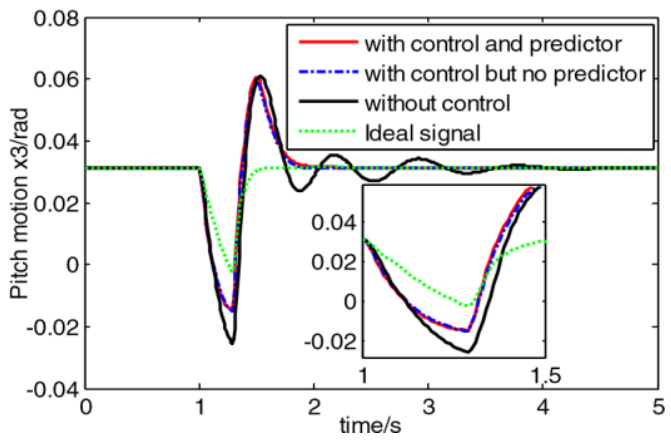

(d) Pitch motion

Fig. 3. Control force simulation for predictors.

Figs. 4-7 are the modeling error which have been bounded via the assumption of LDI based approximation with control via NN model. Furthermore, the assumption of $\|\Delta \Phi(t)\| \leq\left\|\sum_{i=1}^{r} \sum_{l=1}^{r} h_{i}(t) h_{l}(t) \Delta H_{i l} x(t)\right\|$ are satisfied from the illustration in Figs. 4-7.

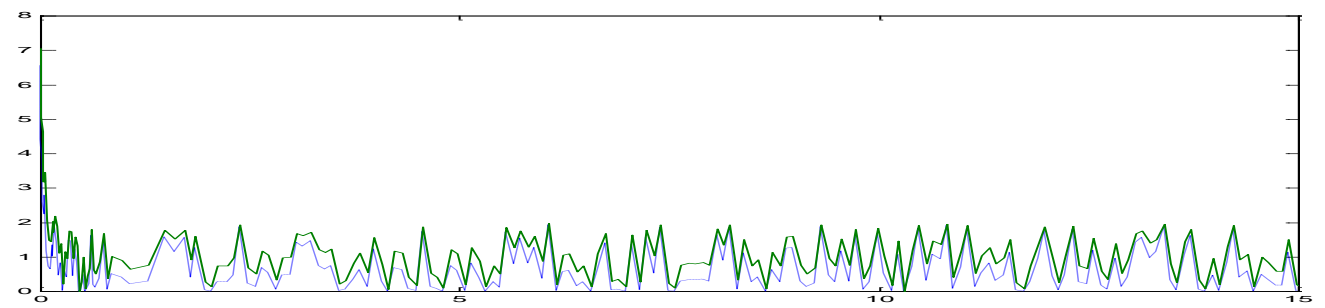


Fig.4. The model error of the vertical displacement of car with control.

Time $(\mathrm{sec})$

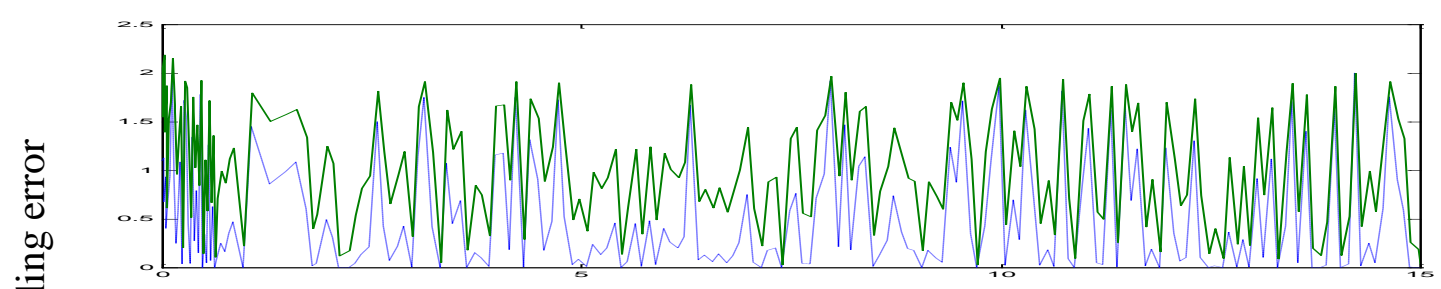

Time $(\mathrm{sec})$

Fig. 5. The model error of pitch motion with control.

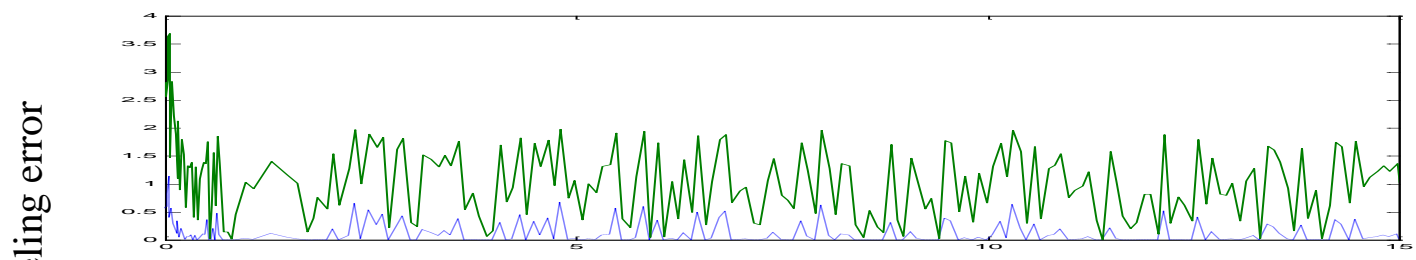

Time (sec)

Fig. 6. The model error of the vertical displacement of front wheel.

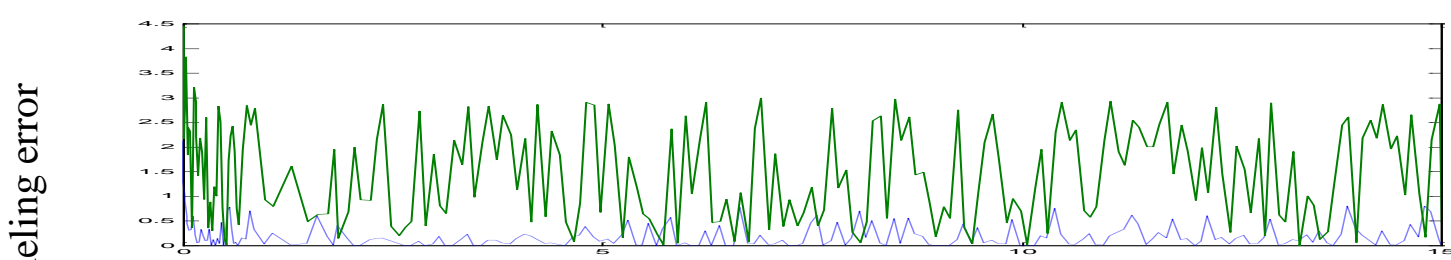

Fig. 7. The model error of the vertical displacement of rear wheel with control.

Time $(\mathrm{sec})$

\section{Conclusion}

This paper describes a new assumption system, demonstrated by the gray model that assisted the LDI neural network, that uses GM $(1,1)$ to predict signals and learns the error of assuming DGM $(2,1)$. They combine the outputs of DGM $(2,1)$ and $\mathrm{NN}$ after time conversion. The simulation results show that the effectiveness of the intelligent prediction system is better than that traditional way. Therefore, it laid a basic foundation for the integration of the active suspension system in the MEW. First, through experimental processes. We considered the nonlinear nature of the active suspension 
and Lyapunov's theory, the law of control for the estimation of ma hardness and damping force parameters and the monitoring of the ideal suspension motion are mentioned. The stability and system probability is proved by Lyapunov such theorem. In addition, the gray DGM $(2,1)$ model is implemented in the controller to assume the suspension movement in advance. The simulation results showed that the proposed method can be without synoptic stabilize the discrete-time nonlinear system. By finding the solution of controlled systems, the advantages of the EBA model also provide flexibility and possibility.

\section{Compliance with Ethical Standards}

The author declares that there are no conflicts of interest regarding the publication of this paper. All analyzed data during this study are included in this article.

\section{References}

Andert, J., Herold, K., Savelsberg, R., \& Pischinger, M. (2017). NVH Optimization of Range Extender Engines by Electric Torque Profile Shaping. IEEE Transactions on Control Systems Technolog ,25,1465-1472.

Chen, C. (2014a). Interconnected TS fuzzy technique for nonlinear time-delay structural systems. Nonlinear Dynamics, 76, 13-22.

Chen, C. (2014b). A criterion of robustness intelligent nonlinear control for multiple time-delay systems based on fuzzy Lyapunov methods. Nonlinear Dynamics, 76, 23-31.

Chen, T., Rao, S., Sabitovich, R.T., Chapron, B., \& Chen, C.Y. (2020). An Intelligent Algorithm Optimum for Building Design of Fuzzy Structures. Iranian Journal of Science and Technology, Transactions of Civil Engineering, 44, 523-531.

Chen, T., Babanin, A.V., Muhammad, A., Chapron, B., \& Chen, J.C. (2020). Evolved Fuzzy NN Control for Discrete-Time Nonlinear Systems. J. Circuits Syst. Comput., 29(1), 2050015. https://doi.org/10.1142/S0218126620500152

Chen, T., \& Chen, J.C. (2020). On the Algorithmic Stability of Optimal Control with Derivative Operators. Circuits Syst. Signal Process., 39, 5863-5881.

Chen, T., Kapron, N., \& Chen, J.C. (2020). Using Evolving ANN-Based Algorithm Models for Accurate Meteorological Forecasting Applications in Vietnam. Mathematical Problems in Engineering. DOI10.1155/2020/8179652.

Chen, T., Kau, D.D., Tai, Y.S., \& Chen, C. (2020). LMI based criterion for reinforced concrete frame structures. Advances in concrete construction, 9(4), 407-412.

Chen, T., (2021). Smart structural stability and NN based intelligent control for nonlinear systems, Smart Structures and Systems, 27(6) ,917-926.

Chen, Z.Y., Meng, Y., Wang, R.Y., and Chen, T. (2021). Apply a robust fuzzy LMI control scheme with AI algorithm to civil frame building dynamic analysis, Computers and Concrete, 28 (4) 433-440. 
Chen, Z.Y. (2021). Grey Signal Predictor and Evolved Control for Practical Nonlinear Mechanical Systems, The Journal of Grey System 33(1), 156-170.

Chen, Z.Y., Wang, R.Y., Meng, Y., Fu, Q. and Chen, T. (2021). Smart structural control and analysis for earthquake excited building with evolutionary design, Struct. Eng. Mech., 79(2), 131-139. https://doi.org/10.12989/sem.2021.79.2.131.

Chen, T., Crosbie, R.C., Anandkumarb, A., Melville, C., \& Chan, J. (2021). Optimized AI controller for reinforced concrete frame structures under earthquake excitation. Advances in concrete construction, 11(1), 1-9.

Cisija-Kobilica, N., Avdakovic, S., Hivziefendic, J., \& Kobilica, A. (2019). Smart transmission system: a new approach for the fault identification, localization and classification in the power system. The Journal of Engineering Research, 7 (2) , 259-280.

Chen, T., Huang, Y.C., Xu, Z., \& Chen, J.C. (2021). Wind vibration control of stay cables using an evolutionary algorithm. Wind and Structures, 32, 71-80.

Chen, T., Kapronand, N., Hsieh, C., \& Cy Chen, J. (2021). Evolved auxiliary controller with applications to aerospace. Aircraft Engineering and Aerospace Technology. 93(4), 529-543.

Chen, T., Kuo, D., \& Chen, C.Y. (2021). Fuzzy C-means robust algorithm for nonlinear systems. Soft Comput., 25, 7297-7305.

Charfeddine, S., \& Jerbi, H. (2021). A Benchmarking analysis of analytical and advanced nonlinear tracking control techniques. Journal of Engineering Research. 9 (3A), 250-267.

Chen, Z.Y., Wang, R.Y., Meng, Y., Fu, Q. and Chen, T. (2022). Stochastic Intelligent GA Controller Design for Active TMD Shear Building, Struct. Eng. Mech., 80(1).

Chen, Z.Y., Meng, Y., Wang, R.Y., and Chen, T. (2022). Systematic Fuzzy Navier-Stokes Equations for Aerospace Vehicles. Aircraft Engineering and Aerospace Technology. Forthcoming.

Cho, H.R., Kim, S., \& Kim, M. (2017). Multiple quantile regression analysis of longitudinal data: Heteroscedasticity and efficient estimation. J. Multivar. Anal., 155, 334-343.

Deshpande,V.S., Mohan,B., Shendge, P.D.,\& Phadke SB. (2014). Disturbance observer based sliding mode control ofactive suspension systems. Journal of Sound and Vibration. 333(11).2281-2296

Huang, Y., Wang, C., Dang, H., \& Lai, S. (2016). Evaluating Performance of the DGM(2,1) Model and Its Modified Models. Applied Sciences, 6, 73.

Kawamoto, Y., Suda, Y., Inoue, H., \& Kondo, T.(2008). Electro-mechanical suspension system considering energy consumption and vehicle manoeuvre. Vehicle System Dynamics, 46,1053 1063.

Kilicaslan, S. (2018). Control of active suspension system considering nonlinear actuator dynamics. Nonlinear Dynamics ,91,1383-1394.

Moreno Ramírez, C., Tomas-Rodriguez, M., \& Evangelou, S.A. (2018). Dynamic analysis of double wishbone front suspension systems on sport motorcycles. Nonlinear Dynamics ,91, 2347-2368.

Shao, Y., \& Su, H. (2012). On Approximating Grey Model DGM(2,1). AASRI Procedia, 1, 8-13.

Sun, X., Zhang, H., Meng, W., Zhang, R., Li, K., \& Peng, T. (2018). Primary resonance analysis and vibration suppression for the harmonically excited nonlinear suspension system using a pair of symmetric viscoelastic buffers. Nonlinear Dynamics, 94,1243-1265.

Singh, N.M., Pratap, B., \& Swarup, A. (2019). Nonlinear robust observer based adaptive control design for variable speed wind turbine. The Journal of Engineering Research, 7 (3), 258-285. 
Tsai, P., Pan, J., Liao, B., Tsai, M., \& Istanda, V. (2011). Bat Algorithm Inspired Algorithm for Solving Numerical Optimization Problems. Applied Mechanics and Materials, 148-149, 134 137.

Xiangyang, X., Siqi, Z., \& Peng, D. (2017). Engine-start Control Strategy of P2 Parallel Hybrid Electric Vehicle. Materials Science and Engineering ,280 012029 doi:10.1088/1757-899X/280/1/012029

Zhang, H., \& Wang, J. (2017). Active Steering Actuator Fault Detection for an Automatically-Steered Electric Ground Vehicle. IEEE Transactions on Vehicular Technology, 66,3685-3702. 